\title{
Crystallization of Aluminum Chloride in the Hydrochloric Acid Process for Production of Alumina From Clay
}

\author{
Lewis Jesse Clark, Willard D. Hubbard, and James I. Hoffman
}

\begin{abstract}
When roasted clay is treated with dilute hydrochloric acid, a solution is obtained that contains aluminum and other soluble constituents of the clay in the form of chlorides. Crystallization as the hydrated chloride, $\mathrm{AlCl}_{3} \cdot 6 \mathrm{H}_{2} \mathrm{O}$, serves to separate the aluminum from the other soluble chlorides. A process is described for this crystallization on a large scale.
\end{abstract}

\section{Introduction}

In 1946 a hydrochloric acid process for the production of alumina from clay 1 was described, which involved the following steps: (1) roasting the clay at about $700^{\circ} \mathrm{C},(2)$ digesting the roasted product in dilute hydrochloric acid, (3) filtering to separate the insoluble siliceous matter from the solution containing the aluminum and soluble impurities such as iron and alkali salts, (4) concentrating the solution, (5) precipitating the aluminum as the hyclrated chloride from the concentrated solution by adding gaseous hydrogen chloride; (6) removing the crystals of hydrated aluminum chloride, (7) washing the crystals to remove adhering impurities, (8) calcining the hydrated chloride to obtain alumina, and (9) recovering hydrochloric acid from the waste products at the end of the process. The process is outlined in the flow sheet in figure 1.

Alumina of satisfactory purity was obtained, but the precipitation of aluminum chloride hexahydrate $\left(\mathrm{AlCl}_{3} \cdot 6 \mathrm{H}_{2} \mathrm{O}\right)$ by saturating the solution with hydrogen chloride (step 5) resulted in considerable loss of hydrochloric acid. This in turn caused an unreason-

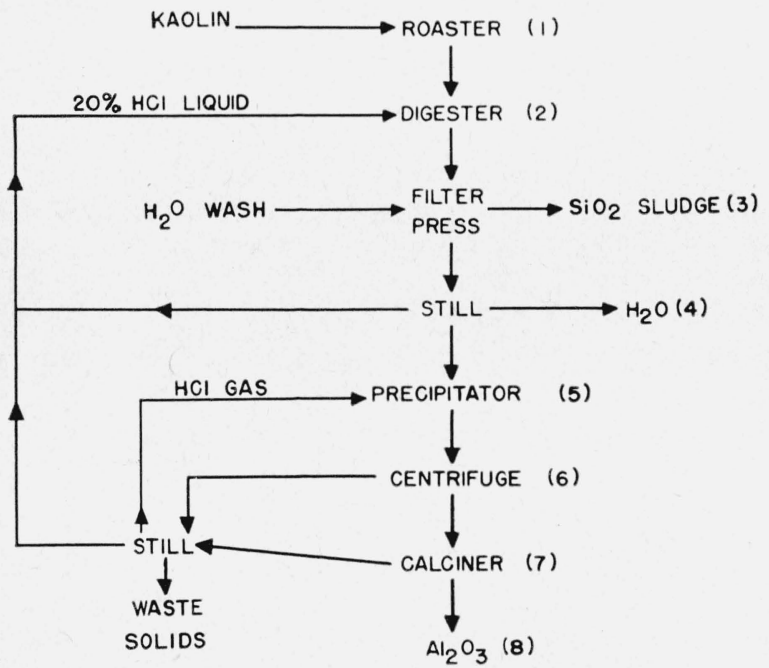

Figure 1. Flow sheet of hydrochloric acid process for the extraction of alumina from clay.

${ }^{1}$ James I. Hoffman, Robert T. Leslie, Harold J. Caul, Lewis Jesse Clark, and John Drake Hoffman, J. Research NBS 3\%, 409 (1946) RP1756. able amount of corrosion with consequent deterioration of equipment. The present discussion is concerned with an improvement of step 5, that is, the separation of the aluminum from the other constituents of clay that are soluble in hydrochloric acid.

In the paper cited, evaporation of the solution until a slurry of crystals formed was mentioned as a means of separating the hydrated aluminum chloride, but during the war, when this work was being done, the necessary equipment was not available. Evaporation with the resultant formation of crystals in hot solution seemed especially well suited for the precipitation of hydrated aluminum chloridebecause its solubility does not vary much with change in temperature. ${ }^{2}$

\section{Crystallization Without Mechanical Stirring}

For good plant operation it seemed obvious that considerable agitation of the solution would be necessary during distillation and crystallization. To obtain such agitation without mechanical stirring, the evaporator shown in figure 2 was designed. It consisted of a Karbate heat exchanger, $A$, connected to a settling tank, $B$. The solution, which was nearly saturated with respect to aluminum chloride, was pumped into the heat exchanger and settling tank assembly so that the level of the solution was somewhat above the inlet of the settling tank $(C$ in fig. 2). This required about 125 gal in the assembly shown. The solution was then heated by means of steam at gage pressures up to $40 \mathrm{lb} / \mathrm{in}^{2}$. As soon as the steam was turned on, the solution in the Karbate tubes within the steam jacket $(D$ in fig. 2) started to rise, and at the boiling temperature a vigorous circulation resulted.

${ }^{2}$ According to Solubilities of inorganic and metal compounds, 3d ed. I, by Atherton Seidell (D. Van Nostrand Co., 1940) the solubility of hydrated aluminum chloride, $\mathrm{AlCl}_{3} \cdot 6 \mathrm{H}_{2} \mathrm{O}$, in water is $57.2 \mathrm{~g}$ per $100 \mathrm{~g}$ of solution at $25^{\circ} \mathrm{C}$ and $59.6 \mathrm{~g}$ per $100 \mathrm{~g}$ of solution at $100^{\circ} \mathrm{C}$. Approximate determinations in our control laboratory indicated the following solubilities in dilute hydrochloric acid:

\begin{tabular}{|c|c|c|c|}
\hline \multirow{2}{*}{ Temperature } & $\begin{array}{c}\text { Specific gravity } \\
\text { of acid }\end{array}$ & \multicolumn{3}{|c|}{ Solubility } \\
\cline { 3 - 4 } & & \multicolumn{2}{|c|}{} \\
\cline { 2 - 4 }${ }^{\circ} C$ & 1.03 & $g / 100$ g solution & lb/gal \\
25 & 1.03 & 50.4 & 5.6 \\
100 & 1.06 & 41.7 & 6.0 \\
25 & 1.06 & 46.8 & 4.5 \\
100 & & & 5.5 \\
\hline
\end{tabular}




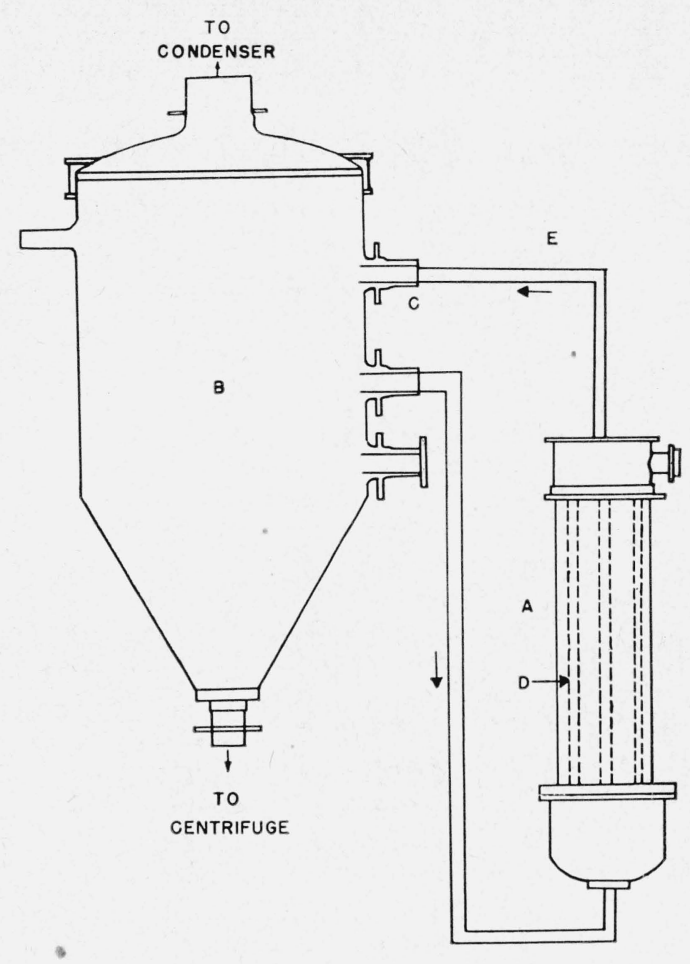

Figure 2. Evaporator without mechanical stirrer.

The boiling point of the solution remained practically constant $\left(124^{\circ} \mathrm{C}\right)$ while crystals were separating. From a knowledge of the volume of the solution in the assembly and of the distillate, together with the concentration of aluminum chloride in the solution in the beginning and at the time of filtration, the approximate weight of crystals could be calculated. With the assembly described, the intention was to evaporate a sufficient volume of acid to produce about $200 \mathrm{lb}$ of the hydrated chloride before separating the crystals from the mother liquor, but an unforeseen difficulty arose. In the glass pipe leading from the heat exchanger to the settling tank ( $E$ in fig. 2 ) bubbles broke, and vapor escaped so rapidly that crystals formed on the inside surface. By the time approximately $100 \mathrm{lb}$ of chloride had separated, this pipe became completely clogged and the distillation had to be discontinued. In addition to the clogging just described, crystals had a tendency to deposit on the gaskets in the pipe lines throughout the system. These deposits on the gaskets resembled doughnuts. If the distillation was continued long enough, the holes in these doughnut-like deposits closed up entirely, and the circulation stopped. In attempts to prevent the formation of these deposits, gaskets, joints, and right angles were entirely eliminated at critical points ( $E$ to $C$ in fig. 2). Pipes of Karbate, Haveg, and glass were selected for smoothness of inside surface, but in all cases the deposits eventually stopped the circulation.

This system of producing crystals was quite satisfactory up to the point where clogging occurred, but because of this difficulty, this evaporator was abandoned in favor of the evaporator described in the next section.

\section{Crystallization With Mechanical Stirring}

The glass-lined steam-jacketed evaporator, shown in figure 3 , is commercially available ${ }^{3}$ and is provided with a motor-driven mechanical stirrer. The nearly saturated solution was pumped into the evaporator, and steam at 10 to $40 \mathrm{lb} / \mathrm{in}^{2}{ }^{2}$ was maintained in the jacket while the solution was mechanically agitated. The approximate quantity of crystals was calculated, as was done in operating the first evaporator. Experience showed that the best slurry for filtering was one that contained 3 to $3.6 \mathrm{lb}$ of aluminum chloride, $\mathrm{AlCl}_{3} \cdot 6 \mathrm{H}_{2} \mathrm{O}$, in a gallon of liquid. In this evaporator no clogging occurred during evaporation. The process is entirely satisfactory if the slurry is cooled and immediately filtered. For best operation, the slurry should not stand overnight because of dense packing of crystals in the bottom of the evaporator and consequent difficulty of starting the flow to the centrifuge.

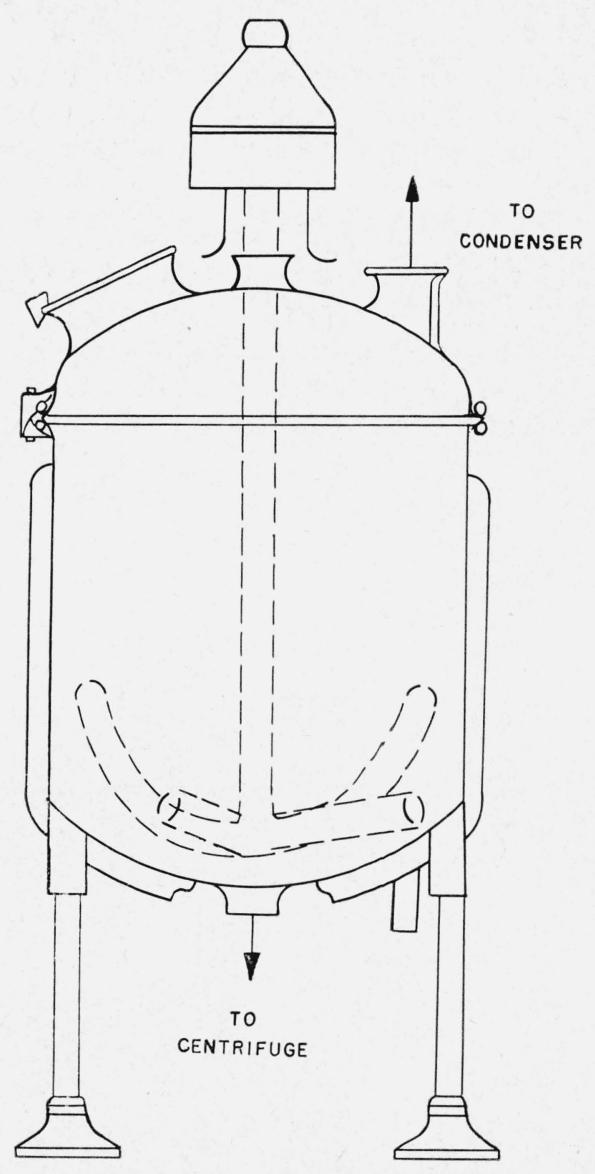

FiguRE 3. Evaporator with a mechanical stirrer.

3 The evaporator used in our plant has a capacity of 100 gal and was purchased from the Pfaudler Co., Rochester, N. Y. Similar equipment can be purchased from Glascote Co., Cleveland, Ohio. 
The acid distillate obtained with this, as well as with the Karbate evaporator, had a specific gravity of about 1.03 .

\section{Separation of the Crystals From the Mother Liquor}

The crystals of aluminum chloride were separated from the mother liquor by means of a rubber-coated centrifuge in the manner described in the original article (see footnote 1). Before filtration, the slurry was cooled to about $40^{\circ} \mathrm{C}$, because rubber-coated equipment deteriorates more rapidly at higher temperatures, and because filtration without preliminary cooling usually results in a glaze over the filter cloth, which slows the flow of the liquid through the cloth. The cooling in both crystallizers was accomplished by cutting off the steam and running tap water through the steam jacket.

The purity of the crystals obtained was about the same as in the process described in the original article.

\section{Cost of Precipitating Aluminum Chloride Hexahydrate}

During the evaporation of the solutions, the steam condensate was collected and the heat input calculated. This value is undoubtedly higher than it should be in good plant operation because the evaporators were not insulated. The results are as follows:

The first evaporator required $16,800,000$ Btu a ton of alumina, equivalent to $\$ 6.75$ on the basis of coal. The second evaporator required 18,336,000 Btu a ton of alumina, equivalent to $\$ 7.36$ on the basis of coal.

In the original process 4,980,000 Btu were required for producing the gaseous hydrogen chloride used in step 5. This was equivalent to $\$ 2.00$ a ton of alumina on the basis of coal at $\$ 6.00$ a ton. In addition, there was a loss of hydrochloric acid in step 5 amounting to $\$ 29.85$ a ton of alumina. The loss of acid in this step is entirely eliminated by the process just described. With the glass-lined evaporator the cost of crystallizing aluminum chloride, equivalent to 1 ton of alumina, is therefore only $\$ 7.36$ as compared with $\$ 31.85$ by the original process $(\$ 29.85$ for loss of acid and $\$ 2.00$ for heat in step 5). This represents a saving of $\$ 24.49$. The total cost for all the steps in the original process is $\$ 109.13$. This improvement in step 5 therefore represents a saving of 22.4 percent. ${ }^{4}$ With reasonable insulation of the equipment the saving may easily be 25 percent.

The authors acknowledge the assistance of Herbert Lowey for his contributions to the mechanical operation of the plant, to George Derbyshire for his assistance in the operation of the Karbate evaporator, and to John Drake Hoffman, who did the laboratory work on which the process is based.

${ }_{4}^{4}$ For original figures on cost, see J. Research NBS 37, 425 (1946) RP1756.

Washington, May 8, 1951 